\title{
Fiber interferometer combining sub-nm displacement resolution with miniaturized sensor head
}

\author{
Lun-Kai Cheng*a, Ronald A.J. Hagen ${ }^{\mathrm{a}}$, Lodi N. Schriek ${ }^{\mathrm{a}}$, Peter M. Toet ${ }^{\mathrm{a}}$, Oana v.d. Togt ${ }^{\mathrm{a}}$ \\ ${ }^{a}$ TNO, Stieltjesweg 1, 2628 CK Delft, The Netherlands
}

\begin{abstract}
The presented interferometer concept enables high-accuracy target displacement measurement in difficult accessible locations and the development of small fiber optic sensor to measure other physical parameters e.g. pressure, vibration, gravity force, etc.. Furthermore, this configuration is basically insensitive to disturbances to the lead fiber between the passive sensor head and the measurement system including the electro-optical parts and the detection interferometer. Two test setups are built and tested to demonstrate the feasibility of high-speed measurement up to $50 \mathrm{kHz}$, low system drift of $\sim 0.5 \mathrm{~nm}$ over $500 \mathrm{~s}$ and a low displacement noise level down to $2.8 \mathrm{pm} / \sqrt{ } \mathrm{Hz}$.
\end{abstract}

Keywords: Fiber optic, interferometer, fiber sensor

\section{INTRODUCTION}

In many fields of operation, high accuracy displacement sensor is required to enable high-precision production or system control, e.g. in the semicon industry and in scientific instruments. Several technologies are available for displacement sensing. Optical sensor has the advantage that for some configurations, the sensing head can be designed to be fully optical and hence not sensitive for Electro-magnetic interference (EMI). Optical interferometer is a well-known technology for high accuracy displacement sensing and is widely used in high-precision equipment, e.g. lithography machines. In comparison to other type of optical displacement sensing technologies e.g. confocal sensor and optical triangulation sensor, interferometer combines sub-nm resolution with potentially large working range which is determined by the coherence length $\mathrm{L}_{\text {coh }}$ of the light source. Conventional interferometers use bulk optical components for beam delivery between the light source and the sensing head. This requires both stable alignments and high mechanical stability of all the parts. In the new generation, optical fibers are used to realize a flexible beam delivery between the light source and the sensor head, containing bulk optical components. A configuration of all fiber optic based interferometer for optical path change measurement is presented in 2005 [1]. High-speed target displacement measurement is demonstrated with sub-nm resolution. In this design, the sensor head consists of an all fiber based interferometer which is connected to the light source and detector using standard single mode fibers. In the all fiber configuration, no critical alignment between the components is required.

In this paper, a configuration of fiber optic interferometer with miniaturized sensor head for displacement measurement is demonstrated in which the fiber optic coupler in the sensor head for beam splitting and recombining is removed. This results in an extremely small and totally passive sensor head for displacement measurement in difficult accessible locations. Another application of this technology is the development of a small sensor which converts the physical parameters to be measured (e.g. pressure or acceleration) to a displacement of the construction in the sensor. The latter can be realized using special GRIN-lens technology/product. Even a GRIN lens with the same diameter as standard singlemode fiber is feasible [2].

\section{FIBER INTERFEROMETER CONFIGURATION}

The main modules of the demonstrated fiber interferometer configuration are shown in Fig. 1. This includes:

- the light source module (1)

- the sensor head (5) with semi reflective mirror and collimating lens and

- $\quad$ the detection interferometer (9).

25th International Conference on Optical Fiber Sensors, edited by Youngjoo Chung, Wei Jin,

Byoungho Lee, John Canning, Kentaro Nakamura, Libo Yuan, Proc. of SPIE Vol. 10323,

1032380 - (C) 2017 SPIE · CCC code: 0277-786X/17/\$18 · doi: 10.1117/12.2265441 


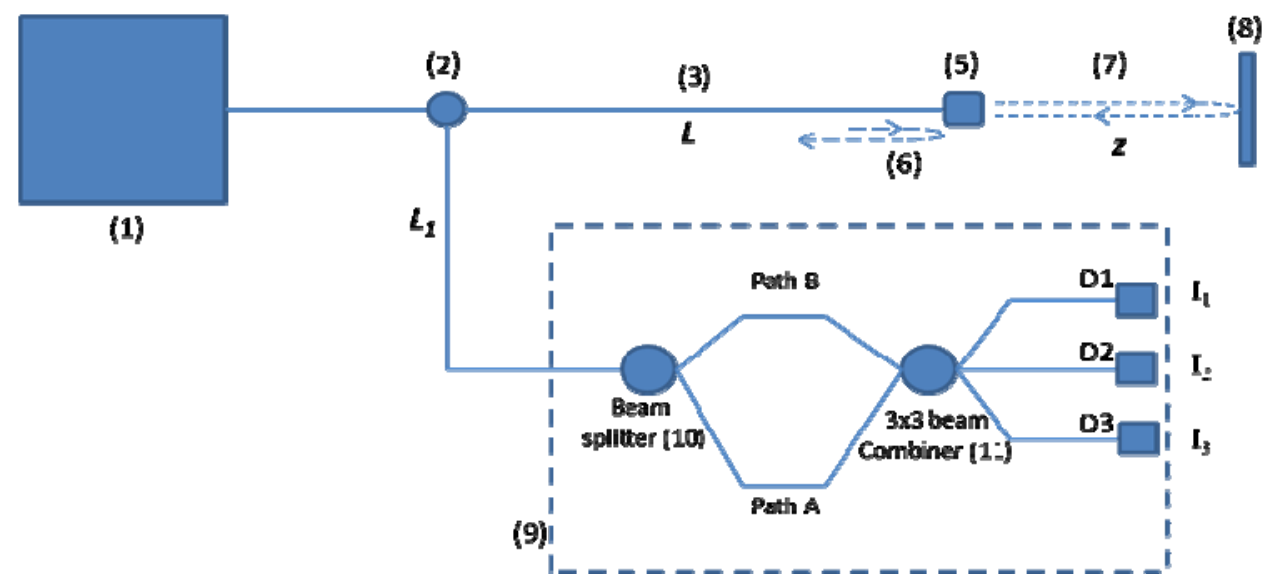

Figure 1. Design of the fiber interferometer.

The light source module operating at the $1550 \mathrm{~nm}$ wavelength window with proper coherence length (1) is connected via an optical circulator (2) and a lead fiber (3) with length $L$ and attenuation $\alpha$ to the sensor head (5). The semi reflective mirror with a reflection coefficient $\mathrm{R}$ in the sensor head generates in reflection the reference beam (6). The fraction $\mathrm{T}$ of the light passing the semi reflective mirror is collimated by a lens in the sensor head to form the sensing beam (7). The target (8) at a distance $\mathrm{z}$ reflects a part of the sensing beam back to the sensor head (5) which couples the sensing beam with an efficiency $\eta$ into the fiber (3). Both the reference beam (6) and the sensing beam (7) are transported by the lead fiber (3) back to the optical circulator (2) and redirect to the detection interferometer (9). The optical path between the optical circulator and the beam splitter (10) of the detection interferometer is $L_{1}$ with attenuation $\alpha_{1}$. For a beam with electric field $\mathrm{E}_{0}$ starting from the optical circulator (2) and travelling through the lead fiber, the electric field $\mathrm{E}_{\text {ref }}$ and $\mathrm{E}_{\text {sens }}$ of the reference beam resp. the sensing beam at the input of the detection interferometer (9) can be described as:

$$
\begin{aligned}
E_{r e f} & =E_{0} e^{i \omega t} \alpha e^{-i k L} R \alpha e^{-i k L} \alpha_{1} e^{-i k L_{1}}=E_{0} \alpha^{2} \alpha_{1} R e^{i\left(\omega t-k\left(2 L+L_{1}\right)\right)}, \\
E_{\text {sens }} & =E_{0} e^{i \omega t} \alpha e^{-i k L} T e^{-2 i k z} \eta T \alpha e^{-i k L} \alpha_{1} e^{-i k L_{1}}=E_{0} \alpha^{2} \alpha_{1} \eta T^{2} e^{i\left(\omega t-k\left(2 L+2 z+L_{1}\right)\right)} .
\end{aligned}
$$

Where $\omega$ is the frequency of the light and $\mathrm{k}=2 \pi / \lambda$ in which $\lambda$ is the wavelength of the light. The spectral bandwidth $\Delta \lambda$ of the light is selected to have a coherence length $\mathrm{L}_{\text {coh }}$ which is much smaller than $\mathrm{z}$ to avoid direct interference between the reference and the sensing beams in the sensor head. In the detection interferometer, the beam splitter (10) splits both the reference beam and the sensing beam with coefficient $C_{A}$ and $C_{B}$ in path $A$ and $B$ respectively. The path length $L_{A}$ is longer than path length $\mathrm{L}_{\mathrm{B}}$. A $3 \times 3$ fiber optic coupler as beam combiner [3] is used to complete the detection interferometer. The $3 \times 3$ fiber optic coupler combines the beams $E_{\text {ref }, A}$ and $E_{s e n s, A}$ in path $A$ with the beams $E_{\text {ref }, \mathrm{B}}$ and $E_{\text {sens,B }}$ in path B. Each beam in path A can interfere with each beam in path B. This results in 4 possible combinations of interference signal. Only the interference with the distance $\mathrm{z}$ in the phase term is of interest for the target displacement measurement. Contribution from other combinations have to be eliminated. This is realized by proper selection of the coherence length $\mathrm{L}_{\text {coh }}\left(=\sim \lambda^{2} / \Delta \lambda\right.$ where $\Delta \lambda$ is the spectral bandwidth of the light source module). Under the conditions $\mathrm{L}_{\text {coh }}<<\mathrm{L}_{\mathrm{A}}-\mathrm{L}_{\mathrm{B}}$ and the difference between $\left(\mathrm{L}_{\mathrm{A}}-\mathrm{L}_{\mathrm{B}}\right)$ and $2 \mathrm{z}$ is in the same order or less than $\mathrm{L}_{\text {coh }}$, only the interference signal with the phase term $\left(L_{A}-L_{B}\right)-2 z$ remains. For a symmetric $3 \times 3$ coupler, the three output interferometric signals become:

$$
\begin{aligned}
& I_{1}=I_{0}+2 V \sqrt{E_{\text {ref }, A 0}^{2} E_{\text {sens }, B 0}^{2}} \cos \left(k\left(\left(L_{A}-L_{B}\right)-2 z\right)\right) \\
& I_{2}=I_{0}+2 V \sqrt{E_{\text {ref }, A 0}^{2} E_{\text {sens }, B 0}^{2}} \cos \left(k\left(\left(L_{A}-L_{B}\right)-2 z\right)+\frac{2 \pi}{3}\right) \\
& I_{2}=I_{0}+2 V \sqrt{E_{\text {ref }, A 0}^{2} E_{\text {sens }, B 0}^{2}} \cos \left(k\left(\left(L_{A}-L_{B}\right)-2 z\right)-\frac{2 \pi}{3}\right)
\end{aligned}
$$


Where $V$ is the visibility of the interference signal and the intensity of $E_{\text {ref }, A}, E_{\text {sens, } A}, E_{\text {ref }, B}$ and $E_{\text {sens, } B}$ are incorporated in $\mathrm{I}_{0}$. In the interferometric signal, both the length $\mathrm{L}$ of the lead fiber and $\mathrm{L}_{1}$ of the connection fiber are common mode and canceled out. Therefore, this system is insensitive to disturbances on the lead fiber. Using the calculation scheme for a symmetric $3 \times 3$ interferometer, the phase $\phi=\mathrm{k}\left(\left(\mathrm{L}_{\mathrm{A}}-\mathrm{L}_{\mathrm{B}}\right)-2 \mathrm{z}\right)$ can be determined from the three interference signals:

$$
\phi=-\arctan \left(\sqrt{3} \frac{I_{1}-I_{2}}{2 I_{3}-I_{1}-I_{2}}\right)
$$

The path length difference $\mathrm{L}_{\mathrm{A}}-\mathrm{L}_{\mathrm{B}}$ in the detection interferometer need to be stabilized by proper mechanical packaging of the detection interferometer for high accuracy calculation of $\phi$ and hence the target distance $z$. The required stability of the wavelength depends on $\left(\mathrm{L}_{\mathrm{A}}-\mathrm{L}_{\mathrm{B}}\right)-2 \mathrm{z}$. For an operational wavelength of $1550 \mathrm{~nm}, \Delta \mathrm{z} / \Delta \phi$ is $123 \mathrm{~nm} / \mathrm{rad}$.

\section{TEST SETUPS AND RESULTS}

Two test setups are built and tested. Setup \#1 was realized with free space bulk optical components for the sensor head to demonstrate and verify the concept. Using commercial GRIN lens components, a miniaturized sensor head is built and tested. This type of sensor head is used in Setup \#2 to demonstrate the drift and the phase noise of this concept.

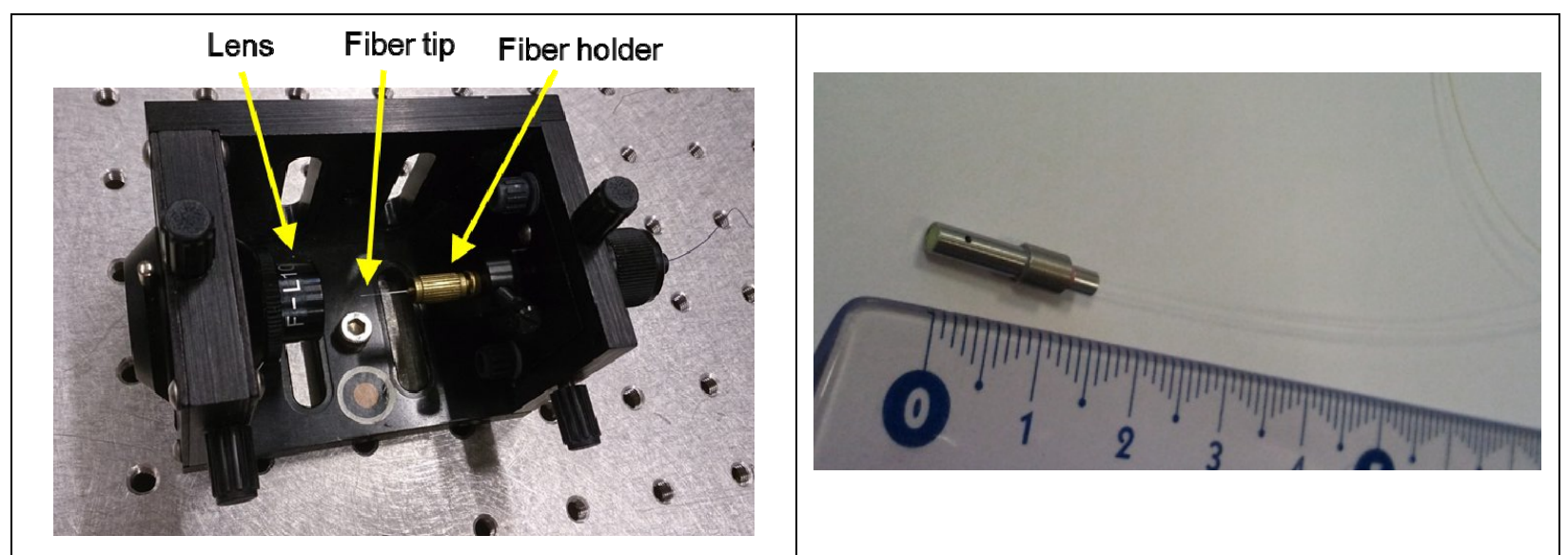

Figure 2. (Left) Sensor head of Setup \#1 using bulk optics. (Right) Miniaturized sensor head in Setup \#2.

\subsection{Setup \#1 for feasibility demonstration}

In Setup \#1 for the feasibility demonstration, the 4\% reflection on the fiber tip is used as the semi reflective mirror to generate the reference beam. The target mirror is mounted on a piezo actuator to generate a $500 \mathrm{~Hz}$ signal with $293 \mathrm{~nm}$ peak-peak displacement. The control voltage of the piezo actuator is reduced in steps of $-10 \mathrm{~dB}$ to $-40 \mathrm{~dB}$. A measurement frequency of $50 \mathrm{kHz}$ is used to demonstrate high-speed measurement. The measured $500 \mathrm{~Hz}$ target displacement as a function the attenuation is shown in Table 1.

Table 1. The measured peak-peak displacement as a function of the attenuation of the piezo control voltage.

\begin{tabular}{|c|c|}
\hline Attenuation & Peak-peak displacement \\
\hline $0 \mathrm{~dB}$ & $293+/-2 \mathrm{~nm}$ \\
\hline$-10 \mathrm{~dB}$ & $95+/-2 \mathrm{~nm}$ \\
\hline$-20 \mathrm{~dB}$ & $29+/-2 \mathrm{~nm}$ \\
\hline$-30 \mathrm{~dB}$ & $11+/-2 \mathrm{~nm}$ \\
\hline$-40 \mathrm{~dB}$ & - \\
\hline
\end{tabular}


The mechanical instability of Setup \#1 using bulk components results in a phase noise of about $+/-0.015$ rad. For the scale factor $\Delta \mathrm{z} / \Delta \phi=123 \mathrm{~nm} / \mathrm{rad}$, this corresponds to a displacement noise of $+/-2 \mathrm{~nm}$. Due to this displacement noise, the target mirror displacement for $-40 \mathrm{~dB}$ attenuation cannot be determined accurately. For the demonstration of the noise level of this interferometer configuration, Setup \#2 with improved mechanical stability is used.

\subsection{Setup \#2 for drift and noise measurement}

The drift and noise level measurement are performed with Setup \#2. The light source module is a combination of an ASE light source with an athermal FBG to minimize wavelength variations. The bandwidth of the FBG is $1.7 \mathrm{~nm}$. A miniaturized sensor head is realized with COTS components to achieve high mechanical stability (see Fig. 2). Both the sensor head and the target are optimized and shielded to prevent thermal induced changes in $\mathrm{z}$. The detection interferometer with $\mathrm{L}_{\mathrm{A}}-\mathrm{L}_{\mathrm{B}}=7.3 \mathrm{~mm}$ is packaged in an aluminum box to isolate both mechanical and thermal disturbances. A 500s drift measurement is performed with a frequency of $20 \mathrm{~Hz}$. The visibility $\mathrm{V}$ of the interference signal is 0.24 . The phase change measured by the interferometer system is shown in Fig. 3(a). For a scale factor $\Delta z / \Delta \phi$ of $123 \mathrm{~nm} / \mathrm{rad}$, the phase change of $\sim 4 \mathrm{mrad}$ over $500 \mathrm{~s}$ showed in Fig. 3(a) corresponds to a drift in the target position measurement of $\sim 0.5$ $\mathrm{nm}$. The FFT spectrum of the measured phase (Fig. 3(b)) shows the phase noise is dominated by the low frequency drift below $\sim 1 \mathrm{~Hz}$. The phase noise level for frequencies $>5 \mathrm{~Hz}$ is flat and equal to $-120 \mathrm{~dB}$ rad. For the calculation of the normalized phase noise per $1 \mathrm{~Hz}$ bandwidth, this number has to be corrected by the frequency bandwidth of $0.002 \mathrm{~Hz}$ for this measurement. The correction factor is $(0.002)^{1 / 2}=27 \mathrm{~dB}$. The normalized phase noise is calculated to be $-120+27=$ $-93 \mathrm{~dB} \mathrm{rad} / \sqrt{ } \mathrm{Hz}$. Using the scale factor of $123 \mathrm{~nm} / \mathrm{rad}$, this corresponds to a normalized displacement noise level of -51 $\mathrm{dB} \mathrm{nm} / \sqrt{ } \mathrm{Hz}(=2.8 \mathrm{pm} / \sqrt{ } \mathrm{Hz})$.

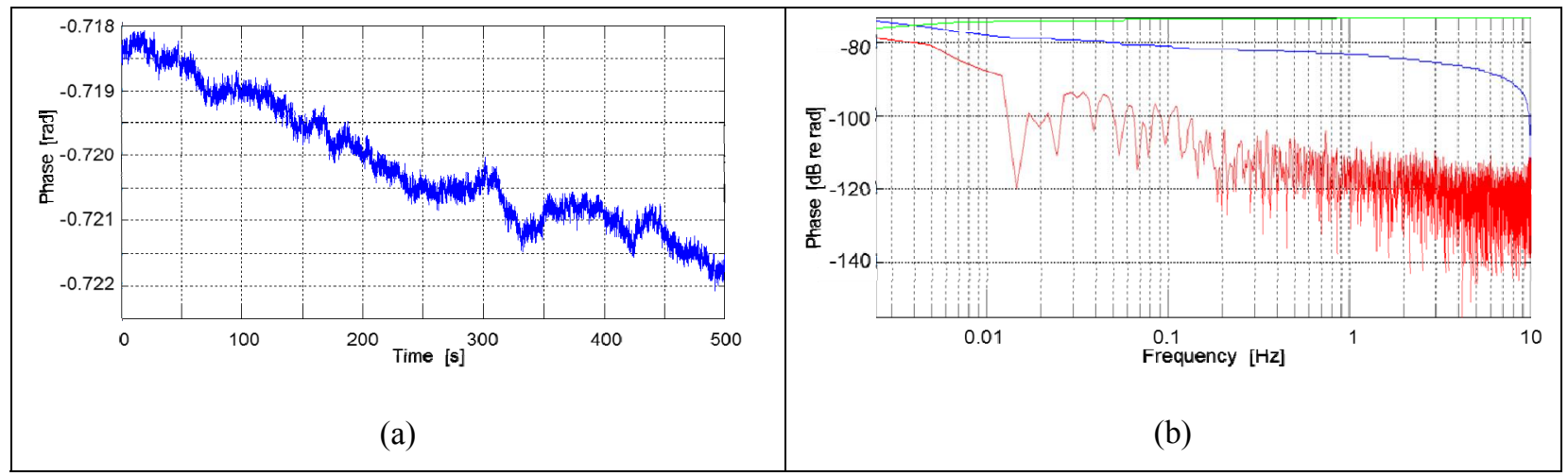

Figure 3. (a) Results of the $500 \mathrm{~s}$ drift measurement. (b) Calculated phase spectrum.

\section{CONCLUSIONS}

The design of a fiber interferometer displacement sensor with a miniaturized sensor head is presented and test setups are built to demonstrate the feasibility of high-speed measurement and high-sensitive performance. This demonstrated displacement sensor with a small passive sensor head can be used in highly demanding applications such as semicon equipment, nuclear fusion installations and for the development of space instruments.

\section{REFERENCES}

[1] Cheng, L. K. et al., "Alternative fringe sensor for DARWIN mission," Proc. SPIE, Techniques and Instrumentation for Detection of Exoplanets II, Vol. 5905, (2005).

[2] SilicaGRIN, http://www.tskg-hd.com/en/service/silicagrin.html

[3] S.K. Sheem et al., "Optical fiber interferometer with $3 \times 3$ directional coupler: Analysis," J. Appl. Phys., Vol. 52, 3865-3872 (1981). 